In-situ probing of lattice response in shock compressed materials using $x$-ray diffraction

J. Hawreliak, M. Butterfield, H. Davies, B. El-Dasher, A. Higginbotham, D. Kalantar, G. Kimminau, J. McNaney, D. Milathianaki, W. Murphy, B. Nagler, H. Lorenzana, N. Park, B. Remington, L. Thorton, T. Whitcher, J. Wark, H. Lorenzana

July 25, 2007

APS Shock compressed condensed matter Kona, HI, United States June 23, 2007 through June 29, 2007 
This document was prepared as an account of work sponsored by an agency of the United States Government. Neither the United States Government nor the University of California nor any of their employees, makes any warranty, express or implied, or assumes any legal liability or responsibility for the accuracy, completeness, or usefulness of any information, apparatus, product, or process disclosed, or represents that its use would not infringe privately owned rights. Reference herein to any specific commercial product, process, or service by trade name, trademark, manufacturer, or otherwise, does not necessarily constitute or imply its endorsement, recommendation, or favoring by the United States Government or the University of California. The views and opinions of authors expressed herein do not necessarily state or reflect those of the United States Government or the University of California, and shall not be used for advertising or product endorsement purposes. 


\title{
IN-SITU PROBING OF LATTICE RESPONSE IN SHOCK COMPRESSED MATERIALS USING X-RAY DIFFRACTION UCRL-PROC-233046
}

\author{
James Hawreliak* $^{*}$, Martin Butterfield* ${ }^{*}$ Huw Davies ${ }^{\dagger}$, Bassem El-Dasher* ${ }^{*}$, Andrew

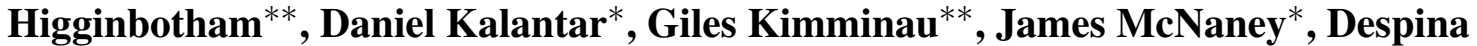

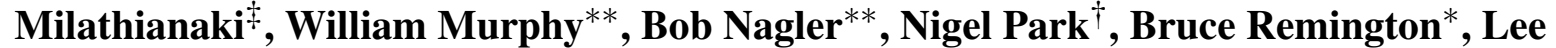 \\ Thorton $^{\dagger}$, Thomas Whitcher**, Justin Wark** and Hector Lorenzana* \\ *Lawrence Livermore National Laboratory, Livermore, CA 94550 \\ ${ }^{\dagger}$ AWE, Aldermaston U.K \\ ${ }^{* *}$ Department of Physics, Clarendon Laboratory, University of Oxford, UK OX1 3PU \\ ¥University of Texas, Austin, TX 78712
}

\begin{abstract}
Lattice level measurements of material response under extreme conditions are required to build a phenomenological understanding of the shock response of solids. We have successfully used laser produced plasma x-ray sources coincident with laser driven shock waves to make in-situ measurements of the lattice response during shock compression for both single crystal and polycrystalline materials. Using a detailed analysis of shocked single crystal iron which has undergone the $\alpha-\varepsilon$ phase transition we can constrain the transition mechanism to be consistent with a compression and shuffle of alternate lattice planes.
\end{abstract}

Keywords: x-ray diffraction, Iron, Experiment, Shock compression

PACS: $62.50 .+\mathrm{p}, 07.35 .+\mathrm{k}, 64.70 . \mathrm{Kb}$

\section{INTRODUCTION}

Building a predictive model of high pressure high strain rate material response which includes elastic compression, plastic deformation, and phase transitions requires a detailed understanding of the governing physical phenomena at the atomic length scale [1]. Techniques such as transmission electron microscopy (TEM) [2], extended xray absorption spectroscopy (EXAFS) [3], and x-ray diffraction (XRD) [4] are all well established diagnostic techniques for studying the microstructure upon recovery of a dynamic loading experiment, or microstructure of materials under static high pressure. Recent developments in these diagnostic techniques, particularly with the advent of short pulse high fluence x-ray and electron sources, have made it possible to make in-situ measurements of dynamic material properties
$[5,6,7]$. We discuss recent developments in in-situ nanosecond $\mathrm{x}$-ray diffraction of crystalline materials.

\section{X-RAY DIFFRACTION DURING SHOCK COMPRESSION IN LASER BASED SYSTEMS}

In-situ dynamic x-ray diffraction (DXRD) provides long scale length structural information about the atomic arrangement at and around the shock front in a non-perturbative manner. DXRD has been key in diagnosing new phases of material under extreme conditions [4]. Short pulse x-rays generated by laser produced plasmas [8], synchrotrons [9], and, in the near future, free electron lasers [10] provide pulses of $\mathrm{x}$-rays short enough to probe lattice response during the shock process. 
A quantitative measurement of lattice response requires the recording of diffraction from regions of shocked and unshocked material. The unshocked measurement gives a fiducial from which the lattice response can be measured. Depending on the laser system, only a single 1 to $2 \mathrm{~ns} \mathrm{X}$-ray pulse is used to backlight the crystal sample. The penetration depth of the x-rays, which depends on both the sample material and the wavelength of $\mathrm{x}$-rays, is typically on the order of several micrometers. This means the x-ray pulse and shock need to be synchronized to within a fraction of a nanosecond to record both static and shocked signals.

For both single crystal and polycrystalline diffraction the approach is basically the same, even though the geometries are very different. In a shocked material the x-rays have some finite penetration depth which allows the probing the shocked and unshocked material with a single $\mathrm{x}$-ray pulse, as illustrated in Fig. 1. In this case we simultaneously get signal from both the compressed material and the uncompressed to compare it with. This is not the case with ramp compression [11].

One advantage of using a laser drive over other types of shock generation techniques is that the laser beam requires very little infrastructure close to the sample to generate a shock, allowing the diffraction to be recorded over a wide range of angles. The shock is generated by the ablation pressure from the expanding plasma from a planar target[12, 13]. Since it is the ablation which drives the pressure pulse the laser beam does not need to be normal to the sample surface. This is useful in allowing diagnostic placement close to the driven surface.

\section{SINGLE CRYSTAL}

Until recently, all dynamic in-situ x-ray diffraction measurements have been performed on single crystals due to their high scattering efficiency. While techniques have been developed to perform diffraction from more complicated polycrystalline materials, single crystal experiments offer the capability to study ideal systems of a single orientation. This also allows direct comparison with molecular dynamics simulations which are currently limited to single crystals [14] or nano-crystalline[15].

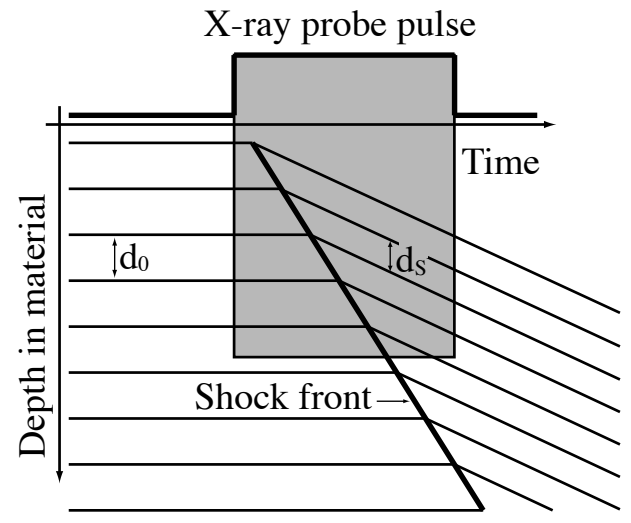

FIGURE 1. A schematic time-depth diagram of x-ray probing of a shocked lattice. The grey block represents the finite temporal pulse length and penetration depth of the $\mathrm{x}$ rays. The horizontal lines represent the lattice spacing in a the material as a function of depth and time.

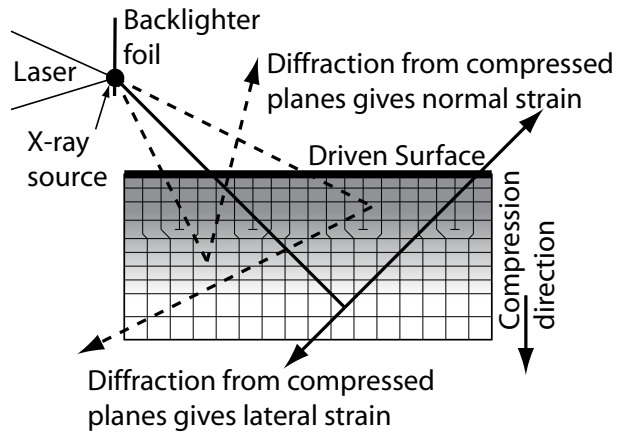

FIGURE 2. A schematic diagram of DXRD from a single crystal using a point source of $\mathrm{x}$-rays. The point source of $x$-rays generates a wide range of incident angles on the sample which diffract where the Bragg condition is met. A change in spacing changes the angle and location where the Bragg condition is satisfied. DXRD can be recorded from planes parallel to the surface, giving axial response, and normal to the surface ( for thin samples), giving plastic response.

\section{TECHNIQUE}

Single crystal x-ray diffraction experiments using similar geometries have been preformed on the Omega lasers system at the University of Rochester 
[16], Janus laser system at the Lawrence Livermore National Laboratory, and Vulcan laser system at the Rutherford Appleton Laboratory [17] . The point source of $\mathrm{x}$-rays is approximately $1 \mathrm{~mm}$ above one side of the driven surface of the sample, as shown in Fig. 2. The X-rays are $\mathrm{K}$-shell radiation of thermal plasma emission generated from a laser of intensity $\approx 10^{14} \mathrm{~W} / \mathrm{cm}^{2}$ incident on a solid foil [8]. The choice of foil determines the required laser intensity and the x-ray photon energy, for example an iron foil will generate $\mathrm{x}$-rays at $1.85 \AA \mathrm{x}$-rays for the $1 \mathrm{~s} 2 \mathrm{p}$ $1 \mathrm{~s}^{2}$ transition when all but two electrons are stripped from each atom. The laser plasma source will emit xrays into $4 \pi$ sterradians, for a pulse length which has nearly the same time duration as the laser pulse [8]. The samples are typically greater than $3 \mathrm{~mm}$ in diameter so the range of incident angles on the sample range from $20^{\circ}$ to $70^{\circ}$, where the higher angles are clipped by the backlighter, and the lower angles are limited by the crystal size. Figure 2, shows a sketch of the experimental setup and the relevant diffraction directions. The x-rays generated in the plasma must pass through the backlighter foil itself. This is useful for materials with $\mathrm{Z}$ greater than 17 , as the foil will preferentially allow the He-like $\mathrm{K}$-shell radiation to penetrate through the foil while filtering out higher and lower energy $\mathrm{x}$-rays.

For DXRD from single crystals, we use a wideangle multi-film plane (MFP) detector, that can cover upto $\pi$ of solid angle for a single detector [18]. Two detectors can be used to provide information about the reflected $\mathrm{x}$-ray diffraction, referred to as the Bragg diffraction geometry, and the transmitted $\mathrm{x}$-ray diffraction, referred to as the Laue diffraction geometry. Film or image plates are used in the MFP because the time resolution is given by the length of the X-ray backlighter pulse. The Bragg geometry is used with both thick and thin samples, whereas, the absorption of the x-rays in the material means the Laue geometry is only used for thin samples, or samples with a long absorption length, i.e. low $\mathrm{Z}$ materials. Not all pieces of the MFP are used on every experiment because of physical limitations of the experimental setups.

Diffraction of the X-rays will only occur at a location on the crystal surface where the Bragg condition is satisfied,

$$
2 d_{h k l} \sin \theta=\lambda_{0}
$$

where $d_{k h l}$ is the spacing of the $h k l$ plane and $\lambda_{0}$ is the wavelength of the probe x-rays. For a single crystal each plane will only diffract over a finited region of the crystal surface which is generally small compared to the crystal size, but because each plane will diffract from a different location on the crystal surface, as the Bragg angle and plane orientation are different, large samples are needed to ensure information is gathered from as many diffraction planes as possible.

Figure 3 shows a schematic diagram of diffraction from a single crystal $3 \mathrm{~mm} \times 3 \mathrm{~mm}$ of bcc iron cell size of $2.85 \AA$ oriented with the (001) axis vertically, using iron $\mathrm{K}$-shell $\mathrm{x}$-rays placed $1 \mathrm{~mm}$ above the surface of the crystal $1 \mathrm{~mm}$ from the center. In this example only a subset of the diffraction planes are shown for illustrative purposes. The diffracted regions on the crystal for each plane will be a conic section determined by a cone with its apex at the backlighter location and the opening angle determined by the Bragg angle. Figure 3 shows the case for a basal plane (in this case (002)), where the Bragg condition is satisfied by a circle, and the (101) plane which is not parallel to the surface generating an off centered elliptical pattern. The diffraction from these finite regions on the crystal surface will be recorded as arcs on the film, where the arc location on the film will give information about the plane orientation and the arc curvature will give information about the plane spacing. One consequence of this geometry is that the arcs on the film give spatial resolution on the sample surface. So it is possible to work out the location on the sample responsible for a particular diffraction arc segment by knowing the experimental geometry. Note, in Fig. 3 that the diffraction from the (101) plane is truncated on the film by the finite size of the crystal.

\section{LATTICE LEVEL MEASUREMENTS}

This technique has been shown to be an extremely powerful diagnostic in determining material structure under shock. Most recently it was successful is diagnosing the high pressure $\varepsilon$-phase in iron during the shock process $[19,20]$. While a measurement of phase is a powerful and unique characteristic of this instrument it can be used to provide insight in the mechanism of the $\alpha-\varepsilon$ transition by the orientation of the $\varepsilon$-phase. 


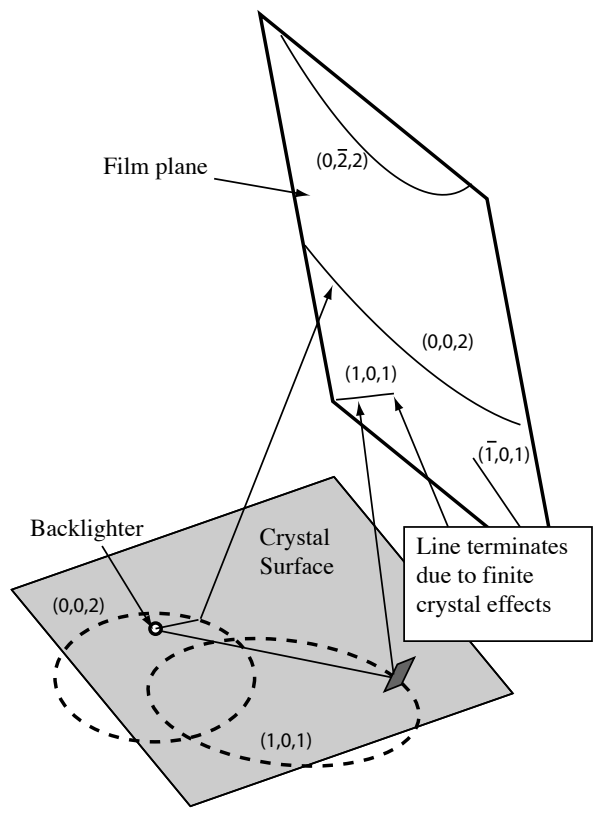

FIGURE 3. A schematic diagram of a point source of x-rays diffracting from a planar crystal surface and the resulting arcs on the film. Diffracted rays from the (002) and (101) planes are identified.

We evaluate the possible degree of rotation allowed within the experimental data and how that corresponds to the three transition mechanisms proposed by Wang and Ingals [21]. Figure 4 shows the diffraction pattern from a $27 \mathrm{GPa}$ shocked single crystal iron which has undergone the phase transition. X-ray diffraction data has shown that the transformed HCP crystal is polycrystalline. Two of the three mechanisms proposed by Wang and Ingals generate a net $5^{\circ}$ rotation of the (2110) HCP planes relative to the equivalent (002) initial BCC planes. Due to the degeneracy of the shift along the $\{110\}_{\mathrm{BCC}}$ family of planes there are 4 variants of the rotation of the $(2 \overline{1} \overline{1} 0)_{\text {HCP }}$ plane, shown in Fig. 4. This significantly over predicts the width of the diffraction lines. By applying a more detailed analysis we can constrain the degree of final rotation of the $\varepsilon$ phase to less than $2^{\circ}$, which is consistent with one of the three posposed transition mechanisms which involves a two step process: 1) compression 2) shuffle.
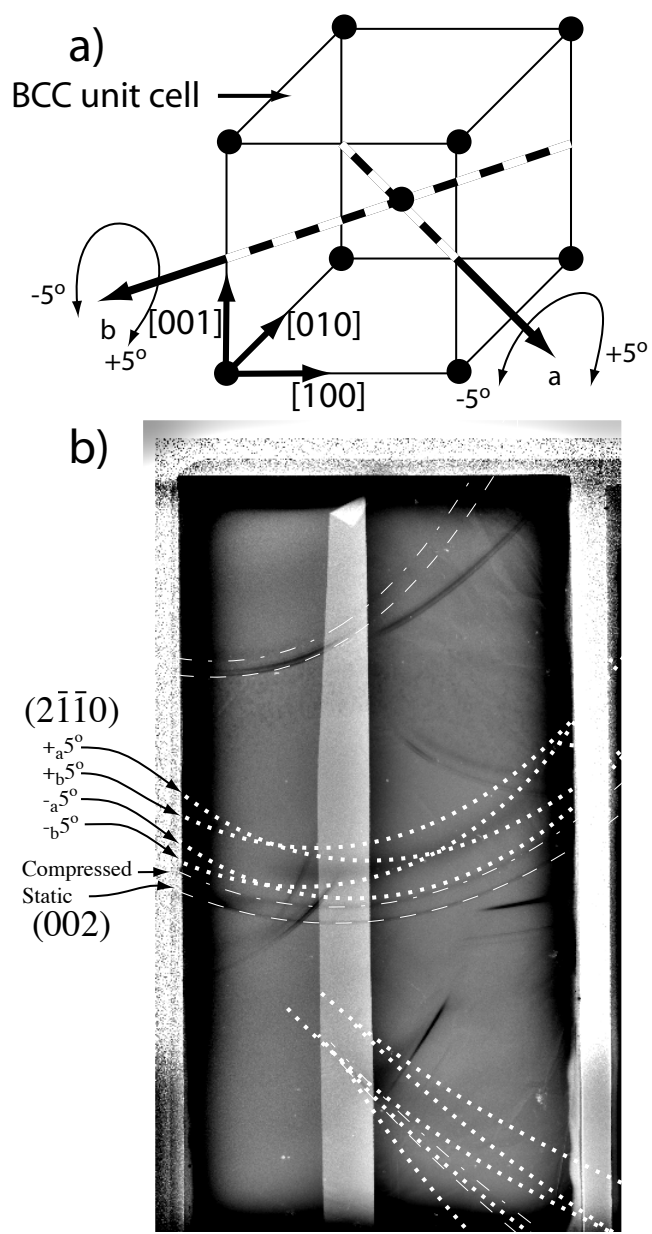

FIGURE 4. Assuming rotation in the transformation mechanism will affect the orientation of the final state. a) shows the four possible rotations due to the degeneracy of the transition pathways, and the knowledge that upon the transition the $\varepsilon$-iron is polycrystalline. b) Attempting to fit experimental data assuming a $5^{\circ}$ rotation upon transformation.

\section{POLYCRYSTALLINE DIFFRACTION}

We have developed a cylindrical pinhole powder camera (CPPC) [22] to study lattice level phenomena in polycrystalline and amorphous materials. The CPPC will allow us to study the response to shock 
of more complex materials that include the influence of grain structure and defects. We briefly discuss the basic principles of this technique and show some DXRD of shock compressed polycrystalline iron.

\section{TECHNIQUE}

The CPPC camera consists of a cylinder of inner diameter $2 r=7 \mathrm{~cm}$, with a sample held at an angle of $i=\pi / 4$ relative to the cylinder axis, $\hat{y}$ as shown in Fig. 5 . An image plate detector covers the whole of the inside surface of the cylinder with holes cut through the image plate so the drive laser beams can access the sample. The camera uses both transmitted and reflected diffraction in a design that is very similar to reflection cameras that have previously been used for texture analysis of static thin films[23]. The foil to be interrogated by diffraction is in the center of the CPPC camera, such that the normal to the rear surface of the foil at the foil center also makes an angle of $\pi / 4$ to the incident $x$-rays. The laser-produced $\mathrm{x}$-ray source is placed $5 \mathrm{~cm}$ from the diffracting foil, on the axis of the cylinder. The position on the image plate detector is defined by $y=r \cot 2 \theta$ to be the distance parallel to the cylinder axis with $y=0$ defined to be at the center of the specimen, and $x=r \phi$ the distance around the circumference of the cylinder. $2 \theta$ is the angle through which the X-rays are scattered with $\theta$ being the standard definition of the Bragg angle, and $\phi$ is the angle between the plane containing the scattered ray and cylinder axis and the plane containing the surface normal to the scattering foil and the cylinder axis.

\section{IRON RESULTS}

We present recent results of in-situ x-ray diffraction from a shock compressed polycrystalline iron foil. A $25 \mu \mathrm{m}$ iron foil was directly ablated with $1.5 \times 10^{15} \mathrm{~W} / \mathrm{cm}^{2}$ of $1.053 \mu \mathrm{m}$ radiation, generating approximately a $11 \mathrm{GPa}$ shock pressure. The timing of the 2 nsec $x$-ray pulse was set to interrogate the material when the shock wave was half way through the sample. By looking at the relative shift of the diffraction lines we can measure the compression of 200 and 211 planes, determined to be $2.2 \% \pm 0.3 \%$ and $1.8 \% \pm 0.2 \%$ respectively for a linear compres-

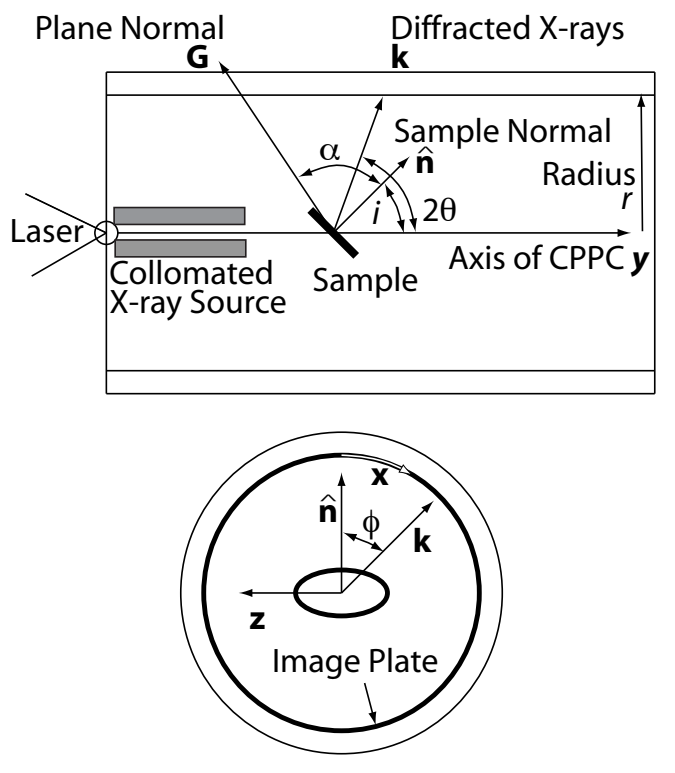

FIGURE 5. Schematic diagram of the CPPC diagnostic

sion of $2 \% \pm 0.2 \%$, see Fig. 6. A more detailed analysis of this data will be presented in a forth coming publication, this data is shown as an example of the capability of the CPPC technique.

\section{DISCUSSION}

We have shown DXRD work for both single and polycrystalline iron. These techniques has already been proven to show insight into atomistic response of shock compressed materials. With the development of simulated x-ray diffraction [24] we can begin directly compare experimental results with molecular dynamics simulations, which suggests the possiblity for making measurements of the microstructure of the material $[25,26]$.

\section{ACKNOWLEDGMENTS}

This work was conducted under the auspices of the U.S. DOE by the UC LLNL and LANL under Contract No. W-7405-Eng-48. The work was supported by LDRD program Project No. 06-SI-004 at LLNL. 


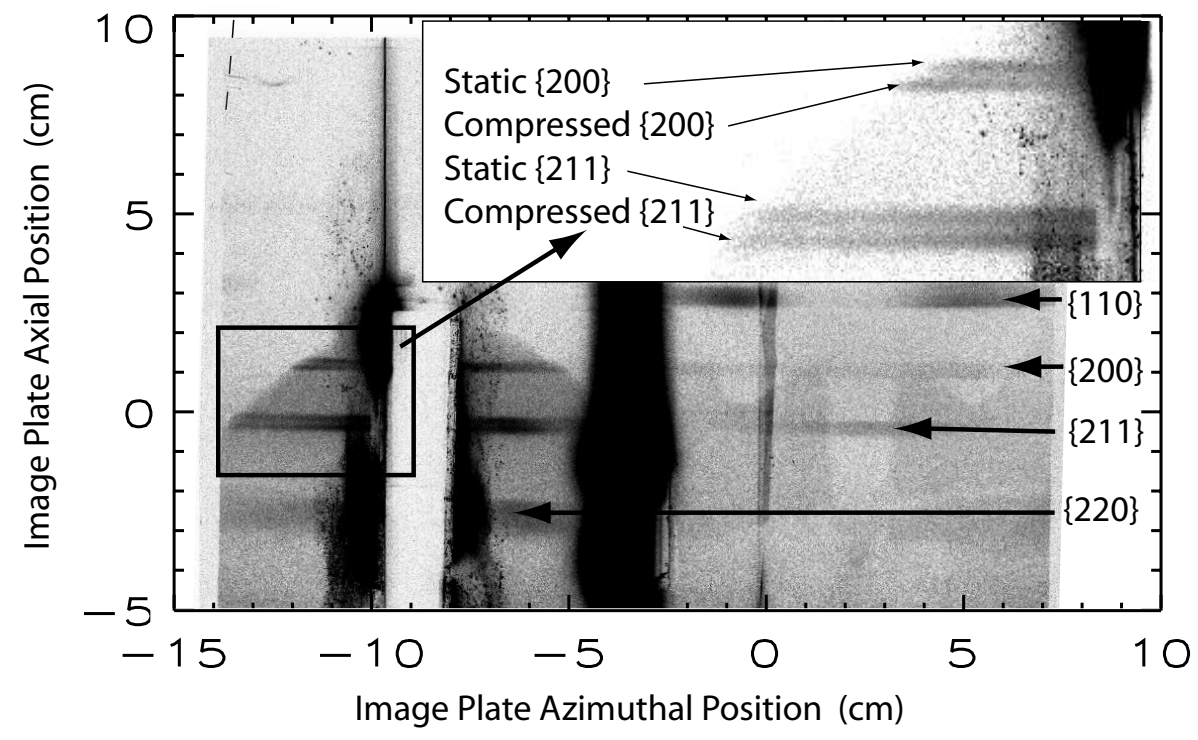

FIGURE 6. Raw image plate data using the CPPC for shock compressed iron foil, $25 \mu \mathrm{m}$ thick. There are four different sets of planes observed, $\{110\},\{200\},\{211\}$, and $\{220\}$ with clear signs of $2 \%$ compression shown on two sets of planes.

\section{REFERENCES}

1. Meyers, M. A., and Meyers, M., Dynamic Behavior of Materials, Wiley-IEEE, 1994.

2. Meyers, M. A. et. al., Acta Materialia, 51, 1211-1228 (2003).

3. Teo, B. K., EXAFS: Basic Principles and Data Analysis, Springer-Verlag, 1986.

4. Mao, H. et. al., Journal of Applied Physics, 38, 272-276 (1967).

5. Loveridge-Smith, A. et. al., Phys. Rev. Lett., 86, 2349-2352 (2001).

6. Yaakobi, B. et. al., Phys. Rev. Lett., 95, 075501 (2005).

7. LaGrange, T. et. al., Appl. Phys. Lett., 89, 044105 (2006).

8. Phillion, D. W., and Hailey, C. J., Physical Review A, 34, 4886-4896 (1986).

9. Schoenlein, R. W. et. al., Applied Physics B: Lasers and Optics, 71, 1-10 (2000).

10. Zholents, A. A., and Fawley, W. M., Phys. Rev. Lett., 92, 224801 (2004).

11. Hawreliak, J. et. al., Astrophysics and Space Science, 307, 285 (2007).

12. Trainor, R. J. et. al., Phys. Rev. Lett., 42, 1154-1157 (1979).

13. Dahmani, F., and Kerdja, T., Physical Review A, 44, 2649-2655 (1991).

14. Holian, B. L. et. al., Physical Review A (General Physics), 22, 2798-2808 (1980).
15. Bringa, E. M. et. al., Science, 309, 1838-1841 (2005).

16. Boehly, T. R. et. al., "The upgrade to the OMEGA laser system," in Proceedings of the tenth topical conference on high temperature plasma diagnostics, AIP, Rochester, New York, 1995, vol. 66, p. 508.

17. Danson, C. N. et. al., Optics Communications, 103, 392-397 (1993).

18. Kalantar, D. et. al., Review of Scientific Instruments, 74, 1929-1934 (2003).

19. Kalantar, D. H. et. al., Phys. Rev. Lett., 95, 075502 (2005).

20. Hawreliak, J. et. al., Physical Review B (Condensed Matter and Materials Physics), 74, 184107 (2006).

21. Wang, F. M., and Ingalls, R., Physical Review B, 57, 5647-5654 (1998).

22. Hawreliak, J. et. al., Review of Scientific Instruments, 78, 083908-6 (2007).

23. Wallace, C. A., and Ward, R. C. C., J. Appl. Crystallogr., 8, 255-260 (1975).

24. Hawreliak, J. et. al., AIP Conference Proceedings, 845, 220-223 (2006).

25. Rosolankova, K. et. al., Journal of Physics: Condensed Matter, 18, 6749-6757 (2006).

26. Bringa, E. M. et. al., Nature Materials, 5, 805-809 (2006). 